\title{
ARCSECOND SCALE POLARIZATION \\ OF BL LAC OBJECTS
}

C. STANGHELLINI ${ }^{1}$, P. CASSARO ${ }^{2}$, M. BONDI ${ }^{1,5}$

AND
D. DALLACASA ${ }^{1,3}$, R. DELLA CECA ${ }^{4}$, R. A. ZAPPALA

${ }^{2}$ Istituto di Radioastronomia CNR - Italy
${ }^{2}$ University of Catania - Italy
${ }^{3}$ JIVE, Dwingeloo - The Netherlands
${ }^{4}$ JHU, Baltimore - USA
${ }^{5}$ NRAL, Jodrell Bank - UK

\section{Introduction}

BL Lac objects are an enigmatic class of active galactic nuclei. They are characterized by high luminosity, a flat radio spectrum that steepens at higher energies, relatively high optical and radio polarization, rapid variability and an optical continuum with weak or absent emission lines (see Urry and Padovani, 1995 for a recent review).

These properties have been interpreted in terms of a relativistic jet closely aligned to the line of sight (Blandford and Rees, 1978, Ghisellini et al., 1993). This model, known as the beaming model, implies that there must be a so called "parent population" of radio sources intrinsically identical to BL Lac objects, but with the jets oriented at large angles to the line of sight. Browne (1983) was the first to propose the low luminosity FR I radio galaxies as the most likely candidates for the "parent population" of the core dominated BL Lac objects. An outcome of the beaming model is that all the properties not depending on orientation should be shared by the BL Lac objects and the FR I radio galaxies.

Prompted by this problem we observed several BL Lac objects from the 1 Jy sample (Stickel at al. 1991) with the VLA and WSRT in order to 

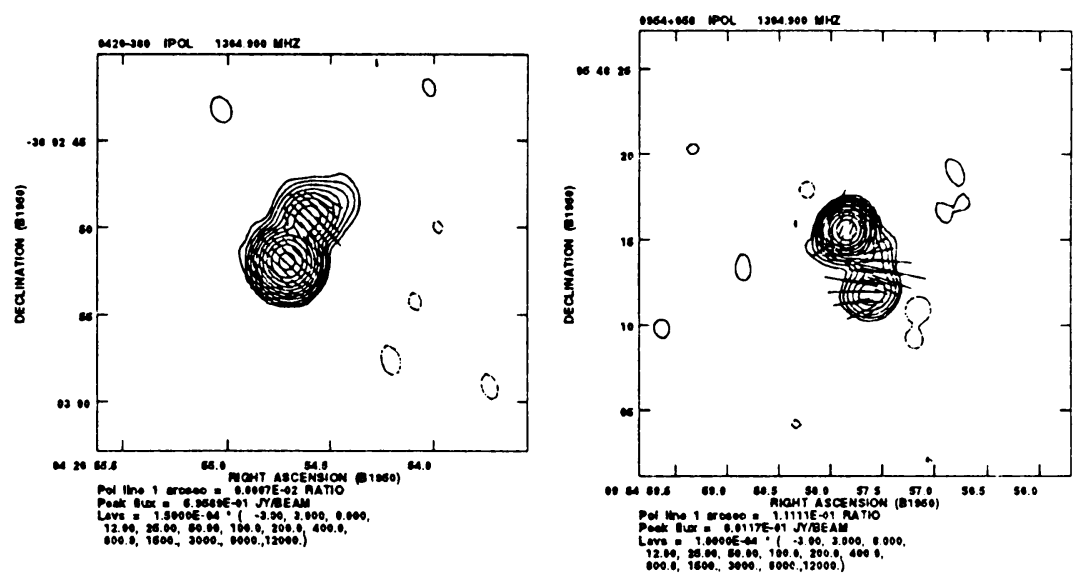

investigate the presence of arcsecond extended emission (see contribution by Bondi et al.) and to study their polarization properties. This aimed to complete the radio information on the complete sample.

\section{Preliminary Results}

The data reduction is still in progress but the results obtained so far are very promising. We detect polarized emission from extended structures reaching and exceeding $40 \%$, while the cores show a fractional polarization between $1 \%$ and $5 \%$. We find several objects where polarized emission is detected along the jet. These can be observed in more detail to study the magnetic field orientation in relation to bends in the jets. This will help in the interpretation of the observed properties in terms of projection effects, jet-ambient interaction and unification models.

An interesting preliminary result is that in some objects (see figures) the $\mathrm{E}$ field of the polarized emission is oriented perpendicular to the jet direction, i.e. the magnetic field structure is basically longitudinal to the jet. This behaviour is commonly seen in the jets of FR II radio sources. The completion of the analysis is necessary to infer statistically significant conclusions in the comparison between the polarization properties of the observed BL Lac objects and the polarization properties of the proposed parent population.

\section{References}

Blandford, R., and Rees, M.J., 1978, in Proc. Pittsburgh Conf. on BL Lac Objects, ed. A.N. Wolfe, p/328.

Browne I.W.A., 1983, MNRAS, 204, 23

Ghisellini, G., et al., 1993, Ap.J., 407, 65.

Stickel M, Padovani P., Urry C.M., Fried J.W., Kühr H, 1991, ApJ, 374, 431

Urry, C.M., and Padovani, P., 1995, PASP, $107,803$. 